\title{
High-efficiency CdTe/CdS core/shell nanocrystals in water enabled by photo-induced colloidal hetero-epitaxy of CdS shelling at room temperature
}

\author{
Hakimeh Zare ${ }^{1}$, Maziar Marandi ${ }^{2}$, Somayeh Fardindoost ${ }^{1}$, Vijay Kumar Sharma ${ }^{3,4}$, Aydan Yeltik ${ }^{3}$, Omid \\ Akhavan $^{1,5}$, Hilmi Volkan Demir ${ }^{3,4}(\triangle)$, and Nima Taghavinia ${ }^{1,5}(\triangle)$ \\ ${ }^{1}$ Institute for Nanoscience and Nanotechnology, Sharif University of Technology, Tehran 14588, Iran \\ ${ }^{2}$ Department of Physics, Faculty of Sciences, Arak University, Arak 38156-8-8349, Iran \\ ${ }^{3}$ Department of Physics, Department of Electrical and Electronics Engineering, and UNAM-Institute of Materials Science and \\ Nanotechnology, Bilkent University, Ankara, Turkey \\ ${ }^{4}$ Luminous! Center of Excellence for Semiconductor Lighting and Displays, Microelectronics Division, School of Electrical and Electronics \\ Engineering, and Physics and Applied Physics Division, School of Physical and Mathematical Sciences, Nanyang Technological \\ University, Singapore 639798, Singapore \\ ${ }^{5}$ Physics Department, Sharif University of Technology, Tehran 14588, Iran
}

Received: 13 October 2014

Revised: 7 February 2015

Accepted: 10 February 2015

(C) Tsinghua University Press and Springer-Verlag Berlin Heidelberg 2015

\section{KEYWORDS}

$\mathrm{CdTe} / \mathrm{CdS}$, core/shell, nanocrystal, thermal stability, photochemical

\begin{abstract}
We report high-efficiency CdTe/CdS core/shell nanocrystals synthesized in water by epitaxially growing $\mathrm{CdS}$ shells on aqueous $\mathrm{CdTe}$ cores at room temperature, enabled by the controlled release of $\mathrm{S}$ species under low-intensity ultraviolet (UV) light illumination. The resulting photo-induced dissociation of $\mathrm{S}_{2} \mathrm{O}_{3}^{2-}$ ions conveniently triggers the formation of critical two-dimensional CdS epitaxy on the CdTe surface at room temperature, as opposed to initiating the growth of individual CdS core-only nanocrystals. This controlled colloidal hetero-epitaxy leads to a substantial increase in the photoluminescence (PL) quantum yield (QY) of the shelled nanocrystals in water (reaching 64\%). With a systematic set of studies, the maximum PL QY is found to be almost independent of the illuminating UV intensity, while the shell formation kinetics required for reaching the maximum QY linearly depends on the illuminating UV intensity. A stability study of the QD films in air at various temperatures shows highly improved thermal stability of the shelled QDs (up to $120^{\circ} \mathrm{C}$ in ambient air). These results indicate that the proposed aqueous $\mathrm{CdTe} / \mathrm{CdS}$ core/shell nanocrystals hold great promise for applications requiring efficiency and stability.
\end{abstract}

Address correspondence to Hilmi Volkan Demir, volkan@bilkent.edu.tr, hvdemir@ntu.edu.sg; Nima Taghavinia, taghavinia@sharif.edu 


\section{Introduction}

Semiconductor quantum dots (QDs) have been utilized in important applications including biological labeling $[1,2]$, light-emitting diodes (LEDs) [3, 4], semiconductor lasers [5], and solar cells [6,7]. The main advantage of QDs is their atom-like band structure that allows for improved luminescence efficiency. Due to technical reasons, III-V QDs are primarily grown using epitaxial methods such as molecular beam epitaxy, while II-VI QDs are commonly synthesized in colloidal forms. There have been numerous demonstrations for the successful use of colloidal QDs in biological applications [8] and optoelectronic applications, including hybrid organic-inorganic LEDs [9-11].

Among the colloidal QDs, CdSe QDs have been the most popular choice for LED studies, particularly in the form of passivated CdSe/ZnS and CdSe/CdS core/shell architectures $[3,12-14]$. The popularity of CdSe QDs stems from their relative stability as well as their high quantum efficiency of luminescence. The synthesis of CdSe QDs usually involves a hot injection process in boiling coordinating solvents. However, this process is not environmentally friendly $[15,16]$. Undesirably, the resulting QD quality is highly dependent on the injection process, making it challenging to perform in a reproducible way unless automated reactors are used.

There are limited reports on the application of CdTe in LEDs [17]. The major reason for its limited application is that it is less stable than CdSe and can be easily oxidized. However, in terms of the energy band positions, CdTe is superior to CdSe. For electrically driven QDs to give strong electroluminescence in a LED, electrons and holes need to be efficiently injected into the QDs. Usually, the major barrier is the hole injection. For many hole-injecting polymers, the position of the highest occupied molecular orbital (HOMO) level is about -5.0 to $-5.5 \mathrm{eV}$ (relative to the vacuum level). The valence band of CdSe lies around $-6.5 \mathrm{eV}$, which creates a barrier of about $1 \mathrm{eV}$ for hole injection. On the other hand, the valence band position of CdTe is desirably around $-5.5 \mathrm{eV}$, which provides a much better HOMO alignment for hole injection. However, this high-positioned valence band undesirably promotes CdTe oxidation [18], which thus necessitates its efficient encapsulation.
Unlike CdSe, CdTe can be grown in aqueous solutions [19]. This is certainly a much easier, more environmentally friendly synthesis method, compared to hot injection organometallic synthesis $[19,20]$. Though highly crystallized CdTe QDs can be conveniently grown using simple chemical precipitation and refluxing methods, they can unfortunately easily be degraded through oxidation. Growing a shell of a more stable semiconductor (such as $\mathrm{CdS}, \mathrm{ZnS}$ and $\mathrm{ZnSe}$ ) around the CdTe cores would thus greatly enhance the stability as well as the efficiency [21-24]. To ensure that the shell coating improves the luminescence efficiency, the shell must be grown epitaxially around the core QD with minimal structural defects at the interface. Otherwise, an efficiency improvement may not be observed despite the shell coating. To avoid structural defects, the lowest lattice mismatch between the core and shell materials is highly favored. For CdTe QDs, the closest match is CdS, which has a 3.6\% lattice mismatch [25]. This should be compared with $\mathrm{ZnS}$, for example, for which the mismatch is $16.5 \%$ [26-28]. Therefore, for CdTe, CdS is one of the most promising capping materials.

There are few previous reports of successful shell formation on CdTe QD cores. Among them, electron beam irradiation (in the megaelectronvolt range) has been employed to coat CdS shells on CdTe QDs without any increase in the QD luminescence quantum yield (QY) [29]. This suggests that the shell crystal quality is not sufficient to fully passivate the cores and simultaneously open defect-induced nonradiative channels, preventing QY enhancement. Shell formation has also previously been reported using microwaveassisted synthesis at relatively high temperatures, giving rise to increased QY [30]. However, this is possible at only high temperatures. A sonochemical method has also been found to enhance the luminescence QY through CdS shell creation, again at relatively high temperatures [31]. Thermochemical methods have also been reported for shelling CdTe QDs at high temperatures [25]. As an alternative, photo-induced methods are also possible. These approaches are very attractive as they are the most effective roomtemperature shelling process. For example, it has been previously reported that prolonged illumination of CdTe QDs (20 days) may improve the luminescence QY [32]. In a faster photo-induced process using high 
intensity lamps, luminescence QY reaching $42 \%$ has been obtained [33]. However, high-intensity illumination causes an inevitable temperature increase, resulting in the presence of both photo-activated and thermally activated processes.

Here, we propose a novel room-temperature shelling route using low-intensity UV illumination enabled by the controlled dissociation of photosensitive $\mathrm{S}_{2} \mathrm{O}_{3}{ }^{2-}$ ions in the aqueous sol. This approach uniquely allows for high-quality epitaxial growth of CdS shells around the aqueous CdTe cores, triggered by the controlled release of $\mathrm{S}$ species, at room temperature. This results in high-efficiency $\mathrm{CdTe} / \mathrm{CdS}$ core/shell nanocrystals (NCs), reaching high QY levels of $64 \%$ in water. This process, however, requires understanding and controlling the kinetics of shell formation. Here, we show that there is a critical point beyond which the crystal quality is not maintained and the improvement of QY is reversed.

In our previous study, we reported that CdS QDs could be conveniently synthesized by the direct photochemical reaction of $\mathrm{CdSO}_{4}$ and $\mathrm{Na}_{2} \mathrm{~S}_{2} \mathrm{O}_{3}$ [34-36]. This previous work demonstrated that the reaction kinetics can be controlled by the irradiation time and intensity. Here, in this work, we show epitaxial-quality shell growth of CdS on CdTe cores to make aqueous highefficiency $\mathrm{CdTe} / \mathrm{CdS}$ core/shell NCs using a completely photo-activated route at room temperature. We find that the maximum PL QY is essentially independent of the illuminating UV intensity. However, the kinetics of shell formation to reach $\mathrm{QY}_{\max }$ linearly depends on the UV intensity. After a critical illumination time, the PL QY declines, which is consistently followed by a steeper red shift and by spectral broadening of the PL peak. After this critical time, the increasing photoluminescence lifetime starts to decrease. The stability study further shows highly improved thermal stability of the shelled QDs. These results are highly promising and encouraging for future applications of $\mathrm{CdTe} / \mathrm{CdS}$ core/shell nanocrystals.

\section{Experimental}

The core CdTe QDs were synthesized in aqueous solution using $\mathrm{NaHTe}$ and $\mathrm{CdSO}_{4}$ as the precursors and thioglycolic acid (TGA) as the capping agent [37].
The NaHTe solution was prepared by a reaction between sodium borohydride and tellurium powder. Briefly, $7.3 \mathrm{mmol}$ of sodium borohydride and $7.0 \mathrm{~mL}$ of deionized (DI) water were transferred to a three-neck flask. Subsequently, $0.87 \mathrm{mmol}$ of tellurium powder (the corresponding molar ratio of $\mathrm{Te} / \mathrm{NaBH}_{4}$ is 0.12 ) was added, and the solution was vigorously stirred for about $3 \mathrm{~h}$ under Ar gas flow. During this time, the dark tellurium powder disappeared, and a white powder of sodium tetraborate precipitated at the bottom of the flask. The clear NaHTe solution was rapidly transferred to $43 \mathrm{~mL}$ of DI water, which was carefully purged with Ar gas.

In parallel, $3.92 \mathrm{mmol}$ of TGA was added to $150 \mathrm{~mL}$ of $\mathrm{CdSO}_{4}$ aqueous solution with $16.0 \mathrm{mM}$ concentration. Then, the solution $\mathrm{pH}$ was adjusted to 9.5 using a few drops of $4 \mathrm{mM} \mathrm{NaOH}$ solution. The freshly prepared $\mathrm{NaHTe}$ solution was injected into the Ar-purged Cd-TGA solution and heated for $600 \mathrm{~min}$ at $100{ }^{\circ} \mathrm{C}$. In the final solution, the molar ratio of Cd:Te:TGA was 2.75:1:4.5. Shortly after injection, the CdTe QDs were formed, increasing in size during the heating process. Finally, the CdTe QDs were precipitated by the addition of acetone, centrifuged, washed several times, and re-dispersed in the initial volume of DI water before the next step.

Photo-induced formation of CdS shell coating on the surface of CdTe QDs was performed as follows. First, $9.0 \mathrm{~mL}$ aqueous solution of $\mathrm{CdSO}_{4}$ and $\mathrm{Na}_{2} \mathrm{~S}_{2} \mathrm{O}_{3}$ with concentrations of 1 and $50 \mathrm{mM}$, respectively, was prepared. Then, $1 \mathrm{~mL}$ of the extracted CdTe QDs with a concentration of $1 \mathrm{mg} / \mathrm{mL}$ was added into this solution, and the $\mathrm{pH}$ was adjusted in the 5-9 range. Finally, the solution was exposed to UV light using a low-pressure UV tube lamp (Philips, $8 \mathrm{~W}$ ) with predominant radiation at $365 \mathrm{~nm}$. The UV light intensity was changed by adjusting the distance between the UV light and reaction vessel. In all tests, a reference sample was prepared by adding 1-mL CdTe NCs into 9.0-mL DI water, which was exposed to the same UV irradiation. The change in absorption and PL spectra of the sample was monitored during the illumination process.

Optical absorption of the QD sols was measured using a Perkin Elmer Lambda $35 \mathrm{UV} /$ Vis spectrophotometer. The PL spectra were recorded using Cary 
Eclipse fluorescence spectrophotometer (Varian) at the excitation wavelength of $360 \mathrm{~nm}$. The time-resolved fluorescence measurements were acquired with a Pico Quant Fluo Time 200 setup using an excitation wavelength of $375 \mathrm{~nm}$. The X-ray diffraction (XRD) patterns were recorded by a Philips X'Pert Pro MPD system. Transmission electron microscopy (TEM) images were recorded using a FEI Tecnai G2F30 microscope. The X-ray photoelectron spectroscopy (XPS) was performed using a monochromatized Al $\mathrm{X}$-ray source and a hemispherical energy analyzer (Spec model EA10). Measurement of the UV light intensity was carried out with a calibrated fiber spectrometer Avantes (AvaSpec-20 48 TEC). The PL QY of QDs was determined by the following relation $[38,39]$

$$
\Phi_{\text {sample }}=\left(\frac{F_{\text {sample }}}{F_{\text {ref }}}\right)\left(\frac{A_{\text {ref }}}{F_{\text {sample }}}\right)\left(\frac{n_{\text {sample }}^{2}}{n_{\text {ref }}^{2}}\right) \Phi_{\text {ref }}
$$

where $F, A$ and $n$ are the integrated fluorescence intensity (area under the emission peak), absorbance at the excitation wavelength, and refractive index of the solvent, respectively. The PL QYs were determined relative to uranine $\left(\mathrm{C}_{20} \mathrm{H}_{10} \mathrm{Na}_{2} \mathrm{O}_{5}\right)$ in water as a reference material (PL QY $=0.92)$. An excitation wavelength of $490 \mathrm{~nm}$ was used for CdTe QDs and uranine.

\section{Results and Discussion}

Figure 1 shows the characteristic features of the core CdTe QDs. The TEM image illustrates crystalline particles with an average size of about $2.94 \mathrm{~nm}$. The absorption and PL of the CdTe QD sol are presented in Fig. 1(b). The absorption edge occurs at about $550 \mathrm{~nm}$.
The complete disappearance of the absorption value for wavelengths larger than $590 \mathrm{~nm}$ demonstrates the lack of scattering loss and the good dispersion of QDs in the sol. The absorption edge corresponds to the QD bandgap energy of $2.25 \mathrm{eV}$ (compared to the bulk bandgap of $1.44 \mathrm{eV}$ ). The QD size can be estimated from the peak absorption wavelength $(530 \mathrm{~nm})$ using an empirical formula [40], which results in a size of $\sim 3 \mathrm{~nm}$, which is well in agreement with the TEM results. The full width at half maximum (FWHM) of the emission is about $46 \mathrm{~nm}$. Fig. 1(c) displays the XRD pattern of the as-synthesized nanocrystals measured with $\lambda_{\text {xray }}$ of $1.54 \AA$. The three major peaks correspond to the (111), (220), and (311) crystalline planes of cubic phase CdTe crystal (JCPDS-150770). The average QD size, estimated using the broadening of the XRD peaks by the Debye-Scherrer formula, is $\sim 2.8 \mathrm{~nm}$, which is in agreement with the size obtained from the TEM and optical measurements.

The core CdTe QDs were shelled using photoinduced growth of CdS in a controlled manner. In the presence of $\mathrm{Cd}^{2+}$ and $\mathrm{S}_{2} \mathrm{O}_{3}^{2-}$ ions under UV illumination, a CdS shell is formed on the CdTe QDs as schematically illustrated in Fig. 2(a). The figure also shows the luminescence color of the shelled CdTe QDs with different illumination times, which show the evolution of emission from green to orange as the illumination time increases. This indicates that CdS shell formation causes a red shift in the luminescence of QDs. The intensity of the UV light was adjusted by changing the distance between the lamp and the sample, and the corresponding change of the intensity with respect to distance is shown in Fig. 2(b). In the log-log curve, we observe a line with a slope of -1 , indicating that
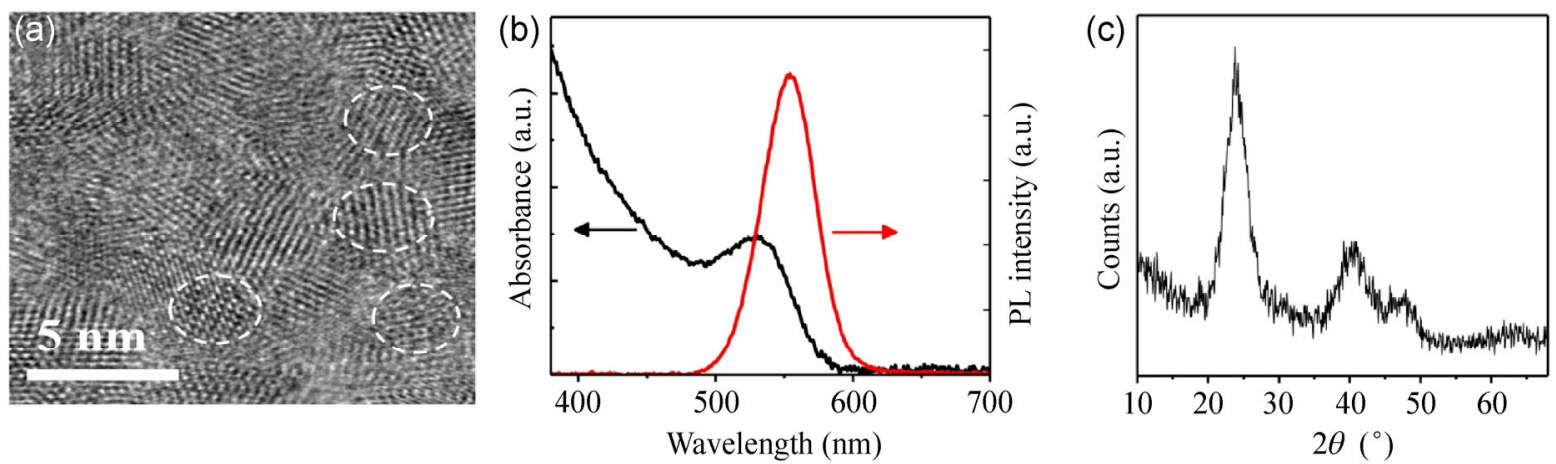

Figure 1 (a) High-resolution TEM (HRTEM), (b) optical absorbance and PL spectra, and (c) XRD pattern of the CdTe NCs prepared by $330 \mathrm{~min}$ of heating at $100{ }^{\circ} \mathrm{C}$.

TSINGHUA 
(a)

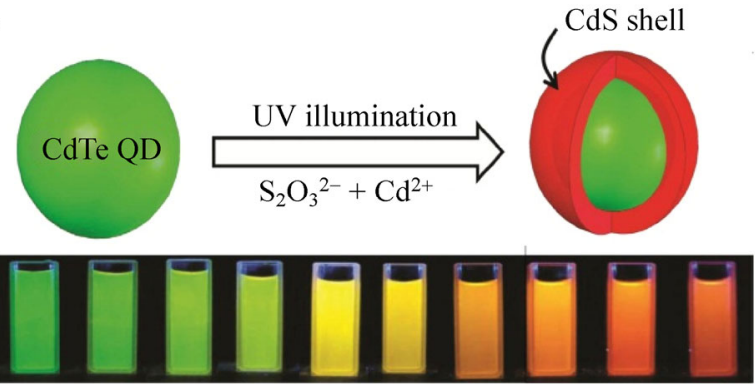

(b)
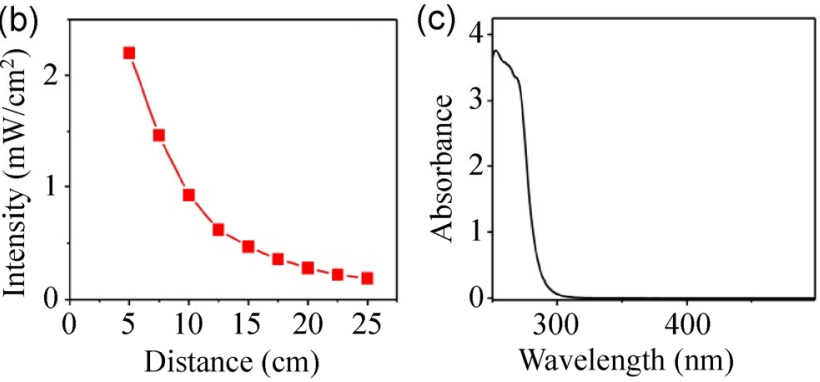

Figure 2 (a) A schematic illustration of the shelling process using the proposed photo-induced shell growth method. The true-color photoluminescence of the core/shell QDs is also shown with increasing illumination time from left to right. (b) The variation of lamp intensity with respect to the lamp distance from the cell. (c) The absorbance of a $\mathrm{Na}_{2} \mathrm{~S}_{2} \mathrm{O}_{3}$ solution of $0.63 \mathrm{M}$.

the intensity is inversely proportional to the distance, which is expected for the cylindrical tube fluorescent lamp. For the highest UV intensity used in the tests $\left(4.4 \mathrm{~mW} / \mathrm{cm}^{2}\right)$, two identical lamps were placed at a distance of $5 \mathrm{~cm}$. Figure 2(c) shows the absorption of $\mathrm{Na}_{2} \mathrm{~S}_{2} \mathrm{O}_{3}$, exhibiting the optical absorption at $365 \mathrm{~nm}$ is very low. Illumination at shorter wavelengths, where the absorption of $\mathrm{Na}_{2} \mathrm{~S}_{2} \mathrm{O}_{3}$ is high, results in direct $\mathrm{CdS}$ QD formation, rather than CdS shell growth. Therefore, illumination with short wavelengths is avoided. In this work, the low absorption at $365 \mathrm{~nm}$ guarantees a two-dimensional (2D) shell growth on the CdTe QDs. This occurs because three-dimensional (3D) growth requires larger kinetic barrier compared to 2D growth; therefore, at low supersaturation conditions, the statistically driven growth of CdS nanoparticles is inhibited, and growth only proceeds on the surface of the existing CdTe QDs. Here, supersaturation is controlled with the low photon flux (lamp intensity) and low absorptivity of $\mathrm{S}_{2} \mathrm{O}_{3}^{2-}$ ions.

To confirm the formation of the CdS shell onto CdTe QDs, TEM, XPS, and XRD techniques were employed as presented in Figs. 3 and 4. The TEM images show
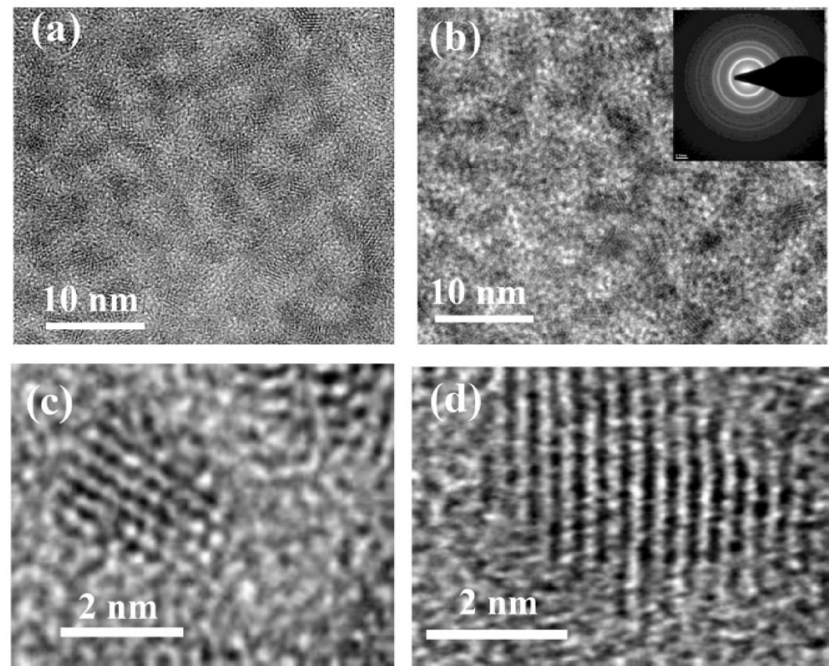

Figure 3 The TEM (top) and HRTEM (bottom) images of (a) and (c) CdTe core QDs, (b) and (d) CdTe/CdS core/shell QDs. The inset image in (b) represents the corresponding SAED diffraction pattern of $\mathrm{CdTe} / \mathrm{CdS}$ core/shell QDs.

an increase in the average QD size after the shelling process, reaching $\sim 4.78 \mathrm{~nm}$ at $180 \mathrm{~min}$ and $\sim 5.65 \mathrm{~nm}$ at $420 \mathrm{~min}$. A clear boundary between the CdTe core and CdS shell is not observed in HRTEM due to the high lattice matching between the CdTe core and CdS shell, indicating that an epitaxial layer is formed and the whole core/shell particles show a single phase [41].

In principle, CdS can also cover the CdTe QDs in the form of CdS nanoparticles, which are already formed in the solution. Here, with this controlled photo-induced shelling process, the $2 \mathrm{D}$ growth on the CdTe surface is strongly favored due to the very low reaction supersaturation. Therefore, we can rule out the formation of $\mathrm{CdS}$ particles on the surface.

Figures 4(a) and 4(b) depict the XPS data showing S2p and Cd3d peaks, respectively, compared with CdTe QDs before and after shell formation. As shown in Fig. 4(a), the sulfur S2p peak is a doublet composed of $S 2 p_{1 / 2}$ and $S 2 p_{3 / 2}$ peaks. For thiol-stabilized CdTe QDs, these peaks are located at the binding energies of $163.4\left(\mathrm{~S} 2 \mathrm{p}_{1 / 2}\right)$ and $162.2 \mathrm{eV}\left(\mathrm{S} 2 \mathrm{p}_{3 / 2}\right)$, separated by $1.2 \mathrm{eV}$. These binding energies confirm the existence of chemical bonds between the TGA ligands and cadmium ions on CdTe QDs surface [42]. For CdTe/CdS core/shell QDs, the S2p peaks are located at the binding energies of $163.0\left(\mathrm{~S} 2 \mathrm{p}_{1 / 2}\right)$ and $161.8 \mathrm{eV}\left(\mathrm{S} 2 \mathrm{p}_{3 / 2}\right)$. This demonstrates an approximately $0.4-\mathrm{eV}$ shift to 
(a)

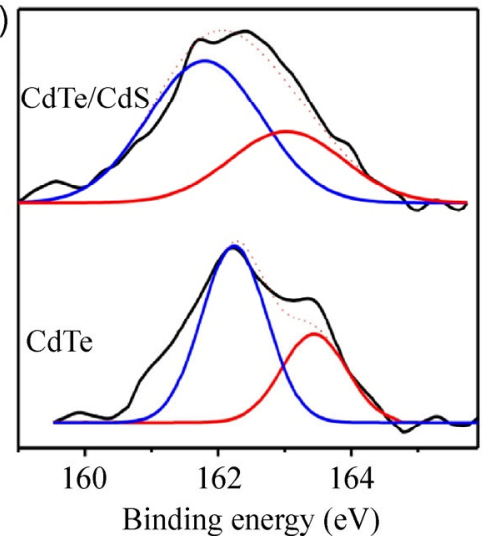

(b)

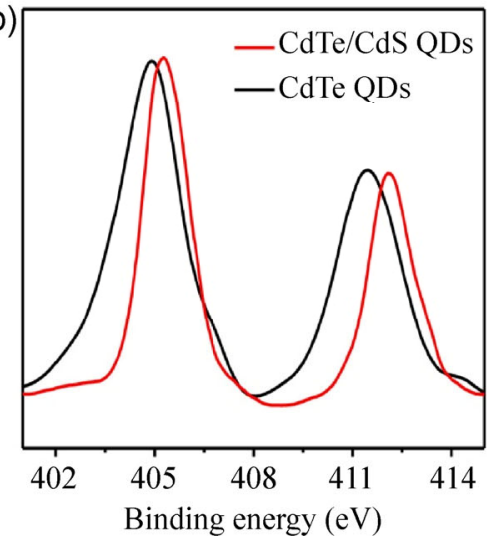

(c)

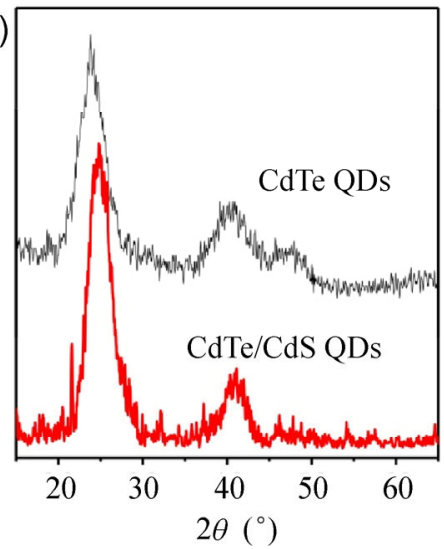

Figure 4 The XPS spectra of CdTe QDs and CdTe/CdS core/shell QDs for the (a) S2p and (b) Cd3d levels. Chemical shift of the S and Cd peaks are observed after shell formation. (c) Comparison of XRD spectra for CdTe core and CdTe core/shell QDs. A shift toward larger angles is observed after shell formation.

lower binding energies for $S 2 \mathrm{p}_{1 / 2}$ and $\mathrm{S} 2 \mathrm{p}_{3 / 2}$, which confirms different coordination of $\mathrm{S}$ atoms on the surface CdTe and CdTe/CdS [32, 42]. This additionally supports the formation of the CdS shell on the surface of CdTe QDs. Figure 4(b) demonstrates the changes in the binding energies of $\mathrm{Cd} 3 \mathrm{~d}$ before and after shelling process. The $\mathrm{Cd} 3 \mathrm{~d}$ peak also appeared as doublet in XPS as $C d 3 d_{1 / 2}$ and $C d_{3} d_{5 / 2}$. For thiol-capped CdTe QDs, the $C d 3 d_{1 / 2}$ and $C_{3} d_{5 / 2}$ peaks are positioned at 411.4 and $404.9 \mathrm{eV}$, respectively. After the shelling process, the peaks are shifted to higher binding energies of 412.1 and $405.3 \mathrm{eV}$, respectively. Because the XPS analysis is surface sensitive, the $\mathrm{Cd}$ binding energy of $\mathrm{CdTe} / \mathrm{CdS}$ QDs is predominately related to the CdS shell (surface) rather than the CdTe core. In contrast, for the CdTe QDs, the binding energy of $\mathrm{Cd}$ corresponds to that of the CdTe core. Therefore, the shift in the Cd binding energy is due to the change in its coordination situation with sulfur or tellurium. This result is also in agreement with the formation of the CdS shell on the surface of CdTe QDs.

Figure 4(c) shows the XRD graphs of the CdTe and CdTe/CdS QDs. Compared with the CdTe QDs peaks, the $\mathrm{CdTe} / \mathrm{CdS}$ QD peaks shift to higher angles toward those of the bulk CdS cubic structure (JCPDS NO. 10-454). This provides additional evidence for the formation of epitaxial layer of CdS on the CdTe core using the proposed room-temperature photo-induced shelling process. The crystal structure of the $\mathrm{CdTe} / \mathrm{CdS}$ NCs remains cubic after the long (420 min) synthesis duration. The SAED diffraction pattern of the sample obtained after 420-min illumination confirms the cubic structure, as shown in Fig. 3(b).

Figure 5(a) presents the time evolution of the absorption and PL spectra as the CdTe/CdS QDs are

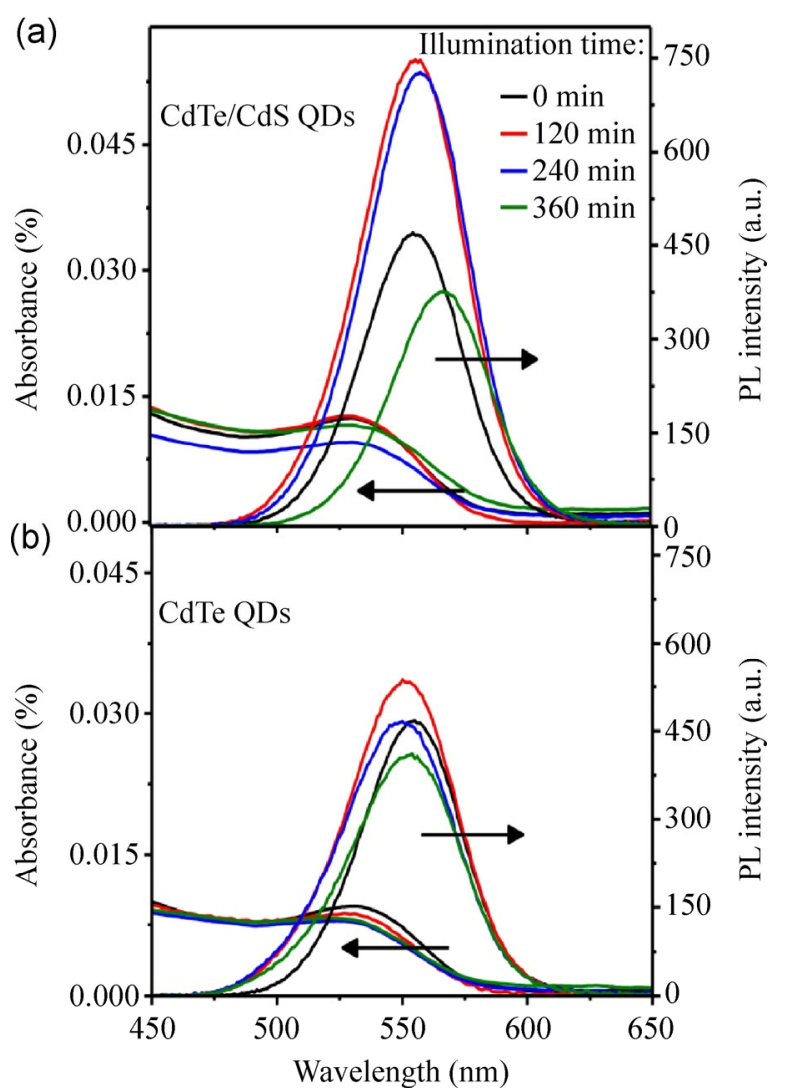

Figure 5 (a) Absorbance and PL spectra of CdTe core (illumination time $=0$ ) and $\mathrm{CdTe} / \mathrm{CdS}$ core/shell QDs prepared with different illumination times. (b) Reference sample containing CdTe QDs in the absence of added $\mathrm{Cd}^{2+}$ and $\mathrm{S}_{2} \mathrm{O}_{3}^{2-}$ ions, illuminated for different times. The UV illumination intensity is $2.2 \mathrm{~mW} / \mathrm{cm}^{2}$. 
formed via $\mathrm{UV}$ illumination in the presence of $\mathrm{Cd}^{2+}$ and $\mathrm{S}_{2} \mathrm{O}_{3}^{2-}$. In this case, the intensity of UV light is $2.2 \mathrm{~mW} / \mathrm{cm}^{2}$. The absorption edge is located at about $555 \mathrm{~nm}$, which does not strongly change over time. The absence of any extra absorption peak at shorter wavelengths may provide evidence that additional CdS nanoparticles were not grown. The PL intensity is more affected by the UV illumination. The PL intensity increases with illumination time up to a certain value and then decreases. The increase of the $\mathrm{PL}$ is the result of effective shell formation on CdTe QD cores, eliminating the surface trap density. On the other hand, the PL peak position shows a red shift. This shift is more clearly observed for larger illumination times. Figure $5(\mathrm{~b})$ shows identical results for the reference samples, which consist of CdTe QDs without the addition of $\mathrm{Cd}^{2+}$ and $\mathrm{S}_{2} \mathrm{O}_{3}^{2-}$ ions. While the optical absorbance is largely unaffected by UV illumination, the PL intensity slightly changes, and the PL peak position shows a slight blue shift. This confirms that the UV-induced shelling process obviously modifies the QD luminescence process.

The change in the PL quantum yield, PL peak position, and PL FWHM during the shelling process is shown in Fig. 6. Figures 6(b), 6(d), and 6(f) show the corresponding comparison data for the reference samples where $\mathrm{Cd}^{2+}$ and $\mathrm{S}_{2} \mathrm{O}_{3}^{2-}$ are not present in the solution. In Fig. 6(a), the QY of the QDs is depicted for different UV illumination times and intensities. The QY values were estimated by comparing the PL of the diluted QD sols with uranine, which has the known QY of $92 \%$. The PL QY is increased with the illumination time up to a maximum value and then starts to decline. This trend occurs more quickly as the $\mathrm{UV}$ intensity is increased. The initial increase in PL QY at short times represents the surface modification of CdTe QDs, resulting in the surface trap reduction and increase in the luminescence intensity. This result also provides supporting evidence for the CdS shell formation over the CdTe QD surface. The long-time decrease of PL QY may be attributed to the formation of a thick CdS shell, which creates a high mismatch with the CdTe core, inducing strain [43]. This strain possibly inhibits the formation of a perfect epitaxial CdS shell and results in nonradiative paths through surface defects.

In Fig. 6(a), the maximum achievable PL QY is observed to be independent of the UV intensity, except for the highest intensity $\left(4.4 \mathrm{~mW} / \mathrm{cm}^{2}\right)$. The maximum PL QY is close to $64 \%$, which is considered to be a
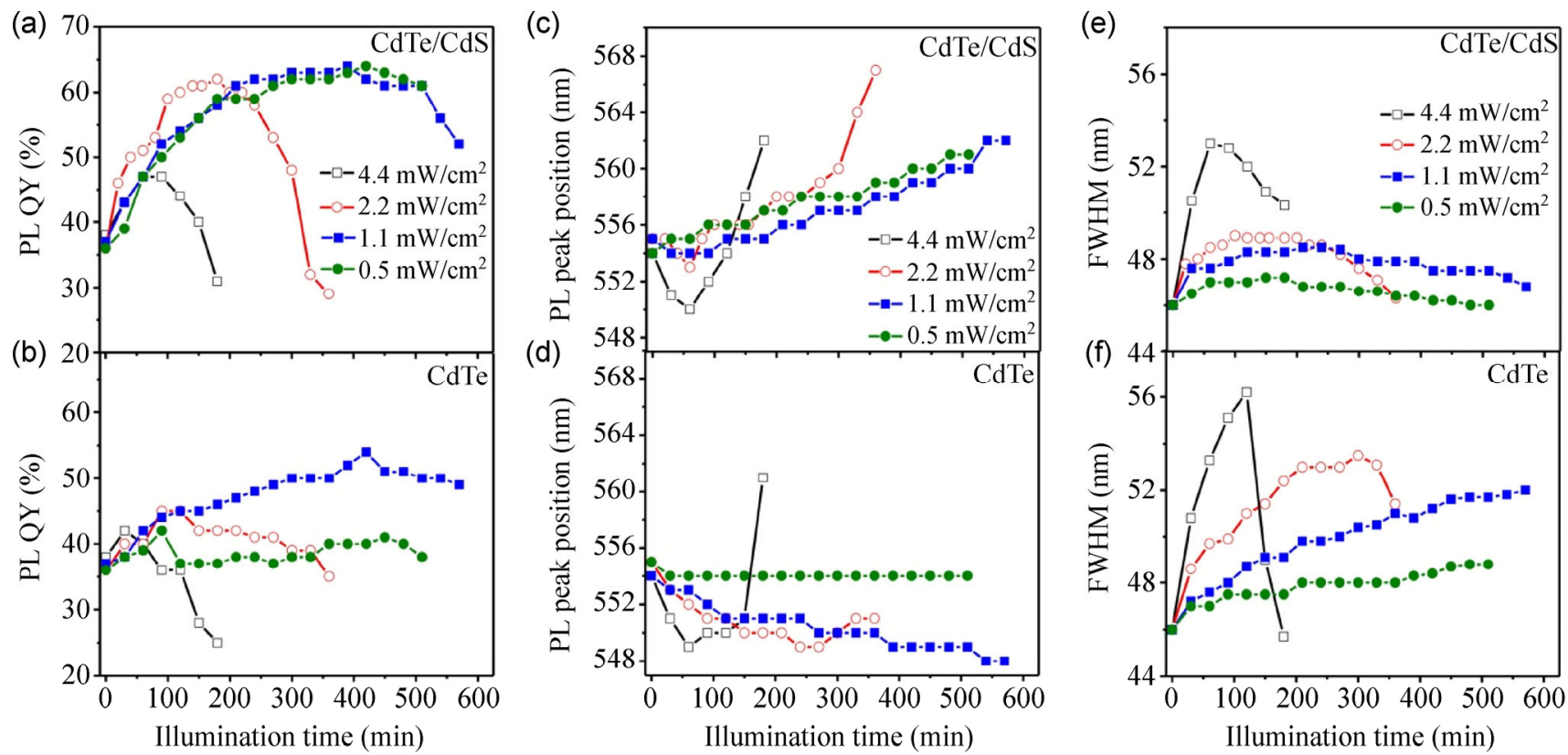

Figure 6 (a), (c), and (e) The change of PL QY, PL peak position, and PL peak FWHM during the formation of CdS shell on CdTe QDs as a function of UV illumination time and for different intensity of UV light. (b), (d), and (f) The same data for reference samples in which $\mathrm{Cd}^{2+}$ and $\mathrm{S}_{2} \mathrm{O}_{3}{ }^{2-}$ ions are not added in the CdTe QD sol. 
very high value for aqueous QDs. The fact that the maximum QY is the same indicates the same quality CdS shell growth over the CdTe cores, independent of the reaction rate (which is controlled by the illumination time). This relationship is not true for the highest illumination intensity $\left(4.4 \mathrm{~mW} / \mathrm{cm}^{2}\right)$, which yields lower QY, that is, lower quality CdS shell. The time required to reach the maximum PL QY is 90,180 , and $360 \mathrm{~min}$ for the UV intensity of $4.4,2.2$ and $1.1 \mathrm{~mW} / \mathrm{cm}^{2}$. For the weakest UV intensity, the maximum QY value does not fall within the measurement time interval.

These maximum values are proportional to the inverse of the illumination intensity, suggesting that the kinetics of the CdS shell formation is a first-order reaction controlled by the light intensity. This result may imply that the shelling process is not limited by the surface reaction. Instead, it is limited by the number of reactive $S$ species produced by the photo-induced dissociation of $\mathrm{S}_{2} \mathrm{O}_{3}^{2-}$ ions, and their migration to the surface of QDs. The production of reactive $\mathrm{S}$ species is proportional to the photon flux, i.e., the light intensity.

Figure $6(\mathrm{~b})$ displays the same data for a reference sample containing CdTe QDs without the addition of $\mathrm{Cd}^{2+}$ and $\mathrm{S}_{2} \mathrm{O}_{3}^{2-}$ ions. Here, we also find photo-induced evolution of the PL intensity. The PL QY of CdTe QDs might be improved by simple light soaking for a long time $[32,42]$. In this case, the effect of illumination intensity is, however, not systematic. In general, the improvement in PL QY is negligible or less than the case when $\mathrm{Cd}^{2+}$ and $\mathrm{S}_{2} \mathrm{O}_{3}^{2-}$ ions are present. This result confirms the critical role of these ions in the photoinduced shell formation.

In Fig. 6(c), the change of PL peak position during the photo-induced process of shell formation is shown. The general trend shows red shift of the PL for CdTe/ CdS QDs and blue shift of PL for the reference CdTe QDs. A red shift for the core/shell CdTe/CdS QDs has been frequently reported as supporting evidence of the shell formation $[16,44,45]$. The reason for this red shift is the type II band alignment of the CdTe/CdS core/shell, which results in partial leakage of the exciton to the CdS shell $[26,43,46]$. Because the conduction band and valence band of $\mathrm{CdS}$ are lower than the CdTe bands, the exciton hole is confined in the core, while the electron leaks into the shell. The leakage of the exciton into the shell corresponds to weaker quantum confinement and a smaller bandgap, hence leading to a red shift in luminescence. Here, the observed peak shift is about $5 \mathrm{~nm}$, which corresponds to $0.02 \mathrm{eV}$. The extent of the red shift is a measure of the shell thickness because, with thicker shells, the exciton radius is expected to increase. The red shift of PL verifies that alloying of $\mathrm{CdS}$ and $\mathrm{CdTe}$ has not occurred because this would form $\mathrm{CdTe}_{1-x} \mathrm{~S}_{x}$, which has a larger bandgap.

The reference samples, which do not contain $\mathrm{Cd}^{2+}$ and $\mathrm{S}_{2} \mathrm{O}_{3}^{2-}$ ions, exhibit PL blue shift with illumination (Fig. 6(d)). The blue shift has been attributed to photoinduced oxidation of unsaturated tellurium atoms at the surface of CdTe QDs, which, in turn, results in the shrinking of particles [31, 32, 41]. As shown in Fig. 6(d), this process is dependent on the illumination intensity. The rate of this "photochemical etching" is proportional to the light intensity (Fig. S2 in the Electronic Supplementary Material (ESM)), which shows that the photo-generated electrons and holes catalyze chemical degradation mechanisms on the surface of QDs.

The effect of UV illumination in improving the PL QY for CdTe/CdS and CdTe QDs is accompanied with an increase of the FWHM of the PL peaks. The increase in FWHM is considerably larger for CdTe QDs compared to CdTe/CdS core/shells, typically $8 \mathrm{~nm}$ versus $3 \mathrm{~nm}$ for $2.2 \mathrm{~mW} / \mathrm{cm}^{2}$ illumination intensity. This increase can be attributed to non-uniformities induced in the shelling process and in photochemical etching for $\mathrm{CdTe} / \mathrm{CdS}$ and CdTe QDs, respectively. This observation of photo-induced spectral broadening is contrary to the previous report that illumination and photoetching results in better mono-dispersity [32].

A rapid deterioration of the PL QY of core/shell QDs is observed for long time illumination, as shown in Fig. 6(a). This rapid QY reduction is accompanied with rapid red shifting of the PL peak and spectral narrowing of the PL peak (Figs. 6(c) and 6(e)). A similar effect is observed for the reference CdTe QDs at high illumination intensity (Figs. 6(b), 6(d), and 6(f)). This sudden change in QDs that occurs after a certain illumination time can be attributed to a kind of re-growth of the existing QDs, which results in larger QDs with red-shifted PL and non-ideal capping. Additional study on the cause of this abrupt change is required, which is planned for future study. 
The luminescence decay time of the shelled QDs is also affected by the UV illumination time. Luminescence intensity was measured at the QD peak emission wavelength for each illumination time. Figure 7 shows the luminescence decay lifetimes for QDs with the shell growth for different reaction times. The presented lifetimes were obtained with the amplitude-averaging of lifetime components [47]. We find a rapid increase in the excited state lifetime with the illumination time and a sharp decrease after a reaction time of about $300 \mathrm{~min}$. The significant increase in lifetimes is a result of the decrease in the electron-hole overlap integral due to the separation of charge carriers in the $\mathrm{CdTe} / \mathrm{CdS}$ QDs. This behavior may not be caused by the increase in the possible competing nonradiative recombination events because we still observe the increase in the steady-state PL intensity. In addition, this continuous increase in lifetimes supports the existence of the inter-transition state, known as quasi type-II, in addition to the surface passivation effects of the shell layer over the core surface. The severe decrease in lifetimes accompanied by the reduced PL intensity after the 300-min reaction time is considered to be associated with the increased nonradiative recombination probability of the electrons, which are confined in the shell region, at the surface defects that are expected to increase with the shell [48]. In addition to the PL intensity, the trends of the PL peak shift and PL FWHM also change after about $300 \mathrm{~min}$ (Fig. 6), indicating that there is an identical cause for these properties. The buildup of stress as the shell thickness increases and shell relaxation through defect formation can explain the changing trends after 300-min illumination.

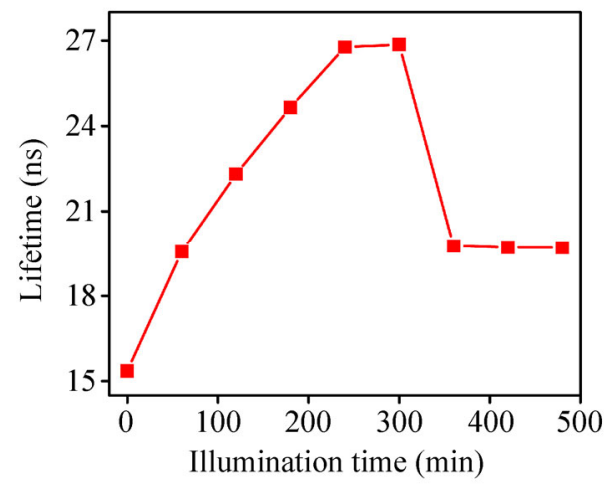

Figure 7 Dependence of the excited-state relaxation time of $\mathrm{CdTe} / \mathrm{CdS}$ QDs on the shell growth reaction time. The UV illumination intensity of $2.2 \mathrm{~mW} / \mathrm{cm}^{2}$ was used for the shell growth.
The growth of an inorganic shell on QDs is known to improve the stability of QDs [26, 44, 46, 49]. Here, the effect of the CdS shell on the thermal stability of QDs was further evaluated by depositing QDs on a glass substrate (the purification of $\mathrm{CdTe} / \mathrm{CdS}$ QDs is not possible by acetone; therefore, the QDs were transferred to a chloroform solvent using cetyltrimethylammonium bromide (CTAB) and before being spin coated on a glass substrate). The PL QY for the solid film samples was measured after $20 \mathrm{~min}$ at different temperatures (in oven). The data is shown in Fig. 8. The PL peak position has been employed as a measure of the CdS shell thickness; PL peaks with a greater red shift correspond to thicker shells. The unshelled CdTe QDs considerably deteriorated at $60^{\circ} \mathrm{C}$ and higher. The stability is improved by the addition of a shell on the QDs. The improvement is dependent on the thickness of the CdS shell. For the thickest shells, the QDs are found to be stable even at $120^{\circ} \mathrm{C}$.

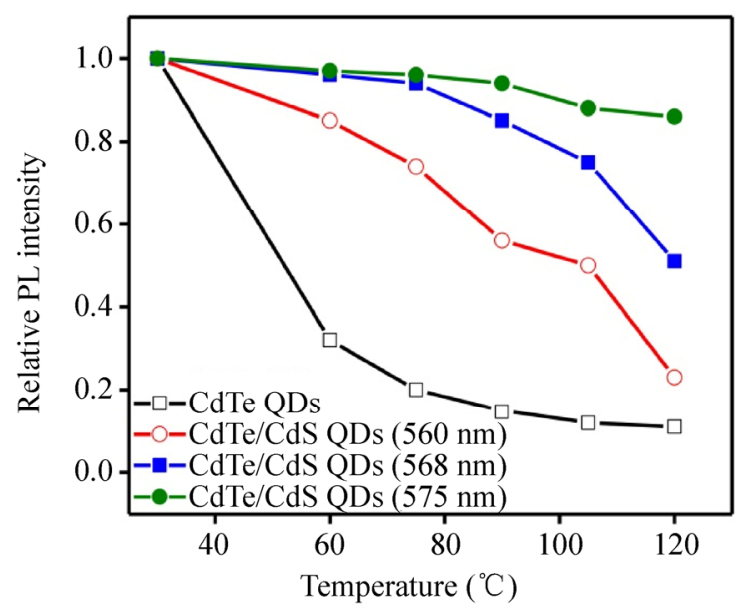

Figure 8 Evaluation of the stability of the core-only and core/ shell QDs, placed in different temperatures for $20 \mathrm{~min}$. The test probe is the normalized PL intensity of QDs. The PL peak position is a measure of the CdS shell thickness; in other words, the peak with a greater red shift is associated with longer illumination time and, hence, thicker CdS shells.

\section{Conclusion}

In this work, we showed the first account of roomtemperature, high-quality, epitaxial shelling of CdS for passivating CdTe cores in water to make systematically high-efficiency aqueous $\mathrm{CdTe} / \mathrm{CdS}$ core/shell nanocrystals using the proposed photo-induced process under low-intensity UV. As we demonstrated from a 
full set of experimental nanocharacterizations, this approach uniquely provides a controllable colloidal hetero-epitaxy process at room temperature to realize $2 \mathrm{D}$ growth of the CdS coating around the CdTe, as opposed to the previous demonstrations of directly growing individual CdS QDs. Here, the time required for the shell formation is relatively short compared to the previously reported routes, and the proposed technique employs low intensity illumination, which guarantees an entirely room-temperature process. The PL QY of the QDs reaches very high levels (up to 64\%) in water, and the shelled QDs show highly improved thermal stability at elevated temperatures (up to $120^{\circ} \mathrm{C}$ ) in ambient air. In principle, this method can be further extended and applied to grow other shell coatings (e.g., ZnS) in water. As a proof of concept demonstration, these results are very encouraging and promising, showing that such aqueous core/shell nanocrystals may be useful for important applications that require efficiency and stability in aqueous media.

\section{References}

[1] Chen, Y. Y.; Liang, H. Applications of quantum dots with upconverting luminescence in bioimaging. J. Photochem. Photobiol. B 2014, 135, 23-32.

[2] Sun, P.; Zhang, H. Y.; Liu, C.; Fang, J.; Wang, M.; Chen, J.; Zhang, J. P.; Mao, C. B.; Xu, S. K. Preparation and characterization of $\mathrm{Fe}_{3} \mathrm{O}_{4} / \mathrm{CdTe}$ magnetic/fluorescent nanocomposites and their applications in immuno-labeling and fluorescent imaging of cancer cells. Langmuir 2010, 26, 1278-1284.

[3] Park, J. Y.; Advincula, R. C. Tunable electroluminescence properties in $\mathrm{CdSe} / \mathrm{PVK}$ guest-host based light-emitting devices. Phys. Chem. Chem. Phys. 2014, 16, 8589-8593.

[4] Lee, K. H.; Lee, J. H.; Song, W. S.; Ko, H.; Lee, C.; Lee, J. H.; Yang, H. Highly efficient, color-pure, color-stable blue quantum dot light-emitting devices. ACS Nano 2013, 7, 7295-7302.

[5] Fafard, S.; Hinzer, K.; Raymond, S.; Dion, M.; McCaffrey, J.; Feng, Y.; Charbonneau, S. Red-emitting semiconductor quantum dot lasers. Science 1996, 274, 1350-1353.

[6] Lan, G. Y.; Yang, Z.; Lin, Y. W.; Lin, Z. H.; Liao, H. Y.; Chang, H. T. A simple strategy for improving the energy conversion of multilayered CdTe quantum dot-sensitized solar cells. J. Mater. Chem. 2009, 19, 2349-2355.

[7] Zhu, H. M.; Yang, Y.; Lian, T. Q. Multiexciton annihilation and dissociation in quantum confined semiconductor nanocrystals. Acc. Chem. Res. 2013, 46, 1270-1279.

[8] Green, M. Semiconductor quantum dots as biological imaging agents. Angew. Chem. Int. Ed. 2004, 43, 4129-4131.

[9] Coe-Sullivan, S.; Woo, W. K.; Steckel, J. S.; Bawendi, M.; Bulović, V. Tuning the performance of hybrid organic/ inorganic quantum dot light-emitting devices. Org. Electron. 2003, 4, 123-130.

[10] Shirasaki, Y.; Supran, G. J.; Bawendi, M. G.; Bulović, V. Emergence of colloidal quantum-dot light-emitting technologies. Nat. Photonics 2012, 7, 13-23.

[11] Molaei, M.; Marandi, M.; Saievar-Iranizad, E.; Taghavinia, N.; Liu, B.; Sun, H. D.; Sun, X. W. Near-white emitting QD-LED based on hydrophilic CdS nanocrystals. J. Lumin. 2012, 132, 467-473.

[12] Mashford, B. S.; Stevenson, M.; Popovic, Z.; Hamilton, C.; Zhou, Z. Q.; Breen, C.; Steckel, J.; Bulovic, V.; Bawendi, M.; Coe-Sullivan, S. et al. High-efficiency quantum-dot light-emitting devices with enhanced charge injection. Nat. Photonics 2013, 7, 407-412.

[13] He, S. J.; Li, S. S.; Wang, F. Z.; Wang, A. Y.; Lin, J.; Tan, Z. A. Efficient quantum dot light-emitting diodes with solution-processable molybdenum oxide as the anode buffer layer. Nanotechnology 2013, 24, 175201.

[14] Qin, H. Y.; Niu, Y.; Meng, R. Y.; Lin, X.; Lai, R. C.; Fang, W.; Peng, X. G. Single-dot spectroscopy of zinc-blende CdSe/ CdS core/shell nanocrystals: Nonblinking and correlation with ensemble measurements. J. Am. Chem. Soc. 2014, 136, 179-187.

[15] Li, H.; Shih, W. Y.; Shih, W. H. Stable aqueous ZnS quantum dots obtained using (3-mercaptopropyl)trimethoxysilane as a capping molecule. Nanotechnology 2007, 18, 495605.

[16] Gu, Z. Y.; Zou, L.; Fang, Z.; Zhu, W. H.; Zhong, X. H. One-pot synthesis of highly luminescent $\mathrm{CdTe} / \mathrm{CdS}$ core/shell nanocrystals in aqueous phase. Nanotechnology 2008, 19, 135604.

[17] Zhou, D.; Liu, M.; Lin, M.; Bu, X. Y.; Luo, X. T.; Zhang, H.; Yang, B. Hydrazine-mediated construction of nanocrystal self-assembly materials. ACS Nano 2014, 8, 10569-10581.

[18] Chin, P. T. K.; Stouwdam, J. W.; van Bavel, S. S.; Janssen, R. A. J. Cluster synthesis of branched CdTe nanocrystals for use in light-emitting diodes. Nanotechnology 2008, 19, 205602.

[19] Gaponik, N.; Talapin, D. V.; Rogach, A. L.; Hoppe, K.; Shevchenko, E. V.; Kornowski, A.; Eychmüller, A.; Weller, H. Thiol-capping of CdTe nanocrystals: An alternative to organometallic synthetic routes. J. Phys. Chem. B 2002, 106, 7177-7185.

[20] Zhang, H.; Wang, L. P.; Xiong, H. M.; Hu, L. H.; Yang, B.; $\mathrm{Li}, \mathrm{W}$. Hydrothermal synthesis for high-quality CdTe nanocrystals. Adv. Mater. 2003, 15, 1712-1715. 
[21] Li, Z.; Dong, C. Q.; Tang, L. C.; Zhu, X.; Chen, H. J.; Ren, J. C. Aqueous synthesis of $\mathrm{CdTe} / \mathrm{CdS} / \mathrm{ZnS}$ quantum dots and their optical and chemical properties. Luminescence 2011, 26, 439-448.

[22] Dai, M. Q.; Zheng, W.; Huang, Z. W.; Yung, L. Y. L. Aqueous phase synthesis of widely tunable photoluminescence emission $\mathrm{CdTe} / \mathrm{CdS}$ core/shell quantum dots under a totally ambient atmosphere. J. Mater. Chem. 2012, 22, 16336-16345.

[23] Tsay, J. M.; Pflughoefft, M.; Bentolila, L. A.; Weiss, S. Hybrid approach to the synthesis of highly luminescent $\mathrm{CdTe} / \mathrm{ZnS}$ and $\mathrm{CdHgTe} / \mathrm{ZnS}$ nanocrystals. J. Am. Chem. Soc. 2004, 126, 1926-1927.

[24] Hewa-Kasakarage, N. N.; Gurusinghe, N. P.; Zamkov, M. Blue-shifted emission in $\mathrm{CdTe} / \mathrm{ZnSe}$ heterostructured nanocrystals. J. Phys. Chem. C 2009, 113, 4362-4368.

[25] Zeng, Q. H.; Kong, X. G.; Sun, Y. J.; Zhang, Y. L.; Tu, L. P.; Zhao, J. L.; Zhang, H. Synthesis and optical properties of type II $\mathrm{CdTe} / \mathrm{CdS}$ core/shell quantum dots in aqueous solution via successive ion layer adsorption and reaction. J. Phys. Chem. C 2008, 112, 8587-8593.

[26] Hines, M. A.; Guyot-Sionnest, P. Synthesis and characterization of strongly luminescing $\mathrm{ZnS}$-capped CdSe nanocrystals. J. Phys. Chem. 1996, 100, 468-471.

[27] Trindade, T.; O'Brien, P.; Pickett, N. L. Nanocrystalline semiconductors: Synthesis, properties, and perspectives. Chem. Mater. 2001, 13, 3843-3858.

[28] Li, L. L.; Chen, Y.; Lu, Q.; Ji, J.; Shen, Y. Y.; Xu, M.; Fei, R.; Yang, G. H.; Zhang, K.; Zhang, J. R. et al. Electrochemiluminescence energy transfer-promoted ultrasensitive immunoassay using near-infrared-emitting CdSeTe/CdS/ZnS quantum dots and gold nanorods. Sci. Rep. 2013, 3, 1529.

[29] Pai, S. C.; Joshi, M. P.; Mohan, S. R.; Deshpande, U. P.; Dhami, T. S.; Khatei, J.; Rao, K. S. K.; Sanjeev, G. Electron irradiation effects on TGA-capped CdTe quantum dots. $J$. Phys. D. Appl. Phys. 2013, 46, 175304.

[30] He, Y.; Lu, H. T.; Sai, L. M.; Su, Y. Y.; Hu, M.; Fan, C. H.; Huang, W.; Wang, L. H. Microwave synthesis of waterdispersed $\mathrm{CdTe} / \mathrm{CdS} / \mathrm{ZnS}$ core-shell-shell quantum dots with excellent photostability and biocompatibility. Adv. Mater. 2008, 20, 3416-3421.

[31] Wang, C. L.; Zhang, H.; Zhang, J. H.; Li, M. J.; Sun, H. Z.; Yang, B. Application of ultrasonic irradiation in aqueous synthesis of highly fluorescent $\mathrm{CdTe} / \mathrm{CdS}$ core-shell nanocrystals. J. Phys. Chem. C 2007, 111, 2465-2469.

[32] Bao, H. B.; Gong, Y. J.; Li, Z.; Gao, M. Y. Enhancement effect of illumination on the photoluminescence of watersoluble CdTe nanocrystals: Toward highly fluorescent CdTe/ CdS core-shell structure. Chem. Mater. 2004, 16, 3853-3859.
[33] Xu, B.; Cai, B.; Liu, M.; Fan, H. S. Ultraviolet radiation synthesis of water dispersed $\mathrm{CdTe} / \mathrm{CdS} / \mathrm{ZnS}$ core-shellshell quantum dots with high fluorescence strength and biocompatibility. Nanotechnology 2013, 24, 205601.

[34] Marandi, M.; Taghavinia, N.; Zad, A. I.; Mahdavi, S. M. Fine tuning of the size of CdS nanoparticles synthesized by a photochemical method. Nanotechnology 2006, 17, 1230-1235.

[35] Marandi, M.; Taghavinia, N.; Zad, A. I.; Mahdavi, S. M. A photochemical method for controlling the size of CdS nanoparticles. Nanotechnology 2005, 16, 334-338.

[36] Taghavinia, N.; Iraji-zad, A.; Mahdavi, S. M.; Reza-esmaili, M. Photo-induced CdS nanoparticles growth. Physica E 2005, 30, 114-119.

[37] Liu, Y. F.; Chen, W.; Joly, A. G.; Wang, Y. Q.; Pope, C.; Zhang, Y. B.; Bovin, J. O.; Sherwood, P. Comparison of water-soluble CdTe nanoparticles synthesized in air and in nitrogen. J. Phys. Chem. B 2006, 110, 16992-17000.

[38] Crosby G. A.; Demas, J. N. Measurement of photoluminescence quantum yields-Review. J. Phys. Chem. 1971, 75, 991-1024.

[39] Talapin, D. V.; Rogach, A. L.; Shevchenko, E. V.; Kornowski, A.; Haase, M.; Weller, H. Dynamic distribution of growth rates within the ensembles of colloidal II-VI and III-V semiconductor nanocrystals as a factor governing their photoluminescence efficiency. J. Am. Chem. Soc. 2002, 124, 5782-5790.

[40] Yu, W. W.; Qu, L. H.; Guo, W. Z.; Peng, X. G. Experimental determination of the extinction coefficient of CdTe, CdSe, and CdS nanocrystals. Chem. Mater. 2003, 15, 2854-2860.

[41] He, Y.; Lu, H. T.; Sai, L. M.; Lai, W. Y.; Fan, Q. L.; Wang, L. H.; Huang, W. Microwave-assisted growth and characterization of water-dispersed $\mathrm{CdTe} / \mathrm{CdS}$ core-shell nanocrystals with high photoluminescence. J. Phys. Chem. B 2006, 110, 13370-13374.

[42] Peng, H.; Zhang, L. J.; Soeller, C.; Travas-Sejdic, J. Preparation of water-soluble $\mathrm{CdTe} / \mathrm{CdS}$ core/shell quantum dots with enhanced photostability. J. Lumin. 2007, 127, 721-726.

[43] Peng, X.; Schlamp, M. C.; Kadavanich, A. V.; Alivisatos, A. P. Epitaxial growth of highly luminescent $\mathrm{CdSe} / \mathrm{CdS}$ core/shell nanocrystals with photostability and electronic accessibility. J. Am. Chem. Soc. 1997, 119, 7019-7029.

[44] Wang, J.; Long, Y. T.; Zhang, Y. L.; Zhong, X. H.; Zhu, L. Y. Preparation of highly luminescent $\mathrm{CdTe} / \mathrm{CdS}$ core/shell quantum dots. Chemphyschem 2009, 10, 680-685.

[45] Reiss, P.; Bleuse, J.; Pron, A. Highly luminescent CdSe/ZnSe core/shell nanocrystals of low size dispersion. Nano Lett. 2002, 2, 781-784. 
[46] Dabbousi, B. O.; Rodriguez-Viejo, J.; Mikulec, F. V.; Heine, J. R.; Mattoussi, H.; Ober, R.; Jensen, K. F.; Bawendi, M. G. (CdSe)ZnS core-shell quantum dots: Synthesis and characterization of a size series of highly luminescent nanocrystallites. J. Phys. Chem. B 1997, 101, 9463-9475.

[47] Lakowicz, J. R. Principles of fluorescence spectroscopy; Springer: New York, 2006.
[48] Smith, A. M.; Mohs, A. M.; Nie, S. M. Tuning the optical and electronic properties of colloidal nanocrystals by lattice strain. Nat. Nanotechnol. 2009, 4, 56-63.

[49] Xie, R. G.; Kolb, U.; Li, J. X.; Basché, T.; Mews, A. Synthesis and characterization of highly luminescent CdSecore $\mathrm{CdS} / \mathrm{Zn}_{0.5} \mathrm{Cd}_{0.5} \mathrm{~S} / \mathrm{ZnS}$ multishell nanocrystals. J. Am. Chem. Soc. 2005, 127, 7480-7488. 